\title{
Erratum to: Noggin Along with a Self-Assembling Peptide Nanofiber Containing Long Motif of Laminin Induces Tyrosine Hydroxylase Gene Expression
}

\author{
Shima Tavakol ${ }^{1,2,3} \cdot$ Sayed Mostafa Modaress Musavi ${ }^{4} \cdot$ Behnaz Tavakol $^{5}$. \\ Elham Hoveizi $^{6}$ - Jafar Ai ${ }^{7,8}$. Seyed Mahdi Rezayat ${ }^{2,9,10}$
}

Published online: 30 August 2016

(C) Springer Science+Business Media New York 2016

Erratum to: Mol Neurobiol

DOI 10.1007/s12035-016-0006-0

The original paper of this article unfortunately contains error. The correct first affiliation of Dr. Shima Tavakol should be written as "Cellular and Molecular Research Center, Iran University of Medical Sciences, Tehran, Iran" instead of "Drug Nanocarriers Research Core, Razi Drug Research Center, Iran University of Medical Sciences, Tehran, Iran."

This change is requested by the author and hereby published.

The online version of the original article can be found at doi:10.1007 /s12035-016-0006-0

Shima Tavakol

tavakol.sh@iums.ac.ir

1 Cellular and Molecular Research Center, Iran University of Medical Sciences, Tehran, Iran

2 Department of Medical Nanotechnology, School of Advanced Technologies in Medicine, Tehran University of Medical Sciences, Tehran, Iran

3 Advance Sciences and Technologies Association, Student's Scientific Research Center, Tehran University of Medical Sciences, Tehran, Iran

4 Shefa Neuroscience Research Center, Khatam al-Anbia Hospital, Tehran, Iran
5 School of Medicine, Kashan University of Medical Sciences, Isfahan, Iran

6 Department of Biology, Faculty of Sciences, Shahid Chamran University of Ahvaz, Ahvaz, Iran

7 Department of Tissue Engineering, School of Advanced Technologies in Medicine, Tehran University of Medical Sciences, Tehran, Iran

8 Brain and Spinal Injury Research Center, Imam Hospital, Tehran University of Medical Sciences, Tehran, Iran

9 Department of Toxicology and Pharmacology, School of Pharmacy, Pharmaceutical Sciences Branch, Islamic Azad University (IAUPS), Tehran, Iran

10 Department of Pharmacology, School of Medicine, Tehran University of Medical Sciences, Tehran, Iran 